\title{
MODELLING OF COMPUTER-ASSISTED MACHINE TOOL VOLUMETRIC VERIFICATION PROCESS
}

\author{
Aguado, S.; Velazquez, J.; Samper, D. \& Santolaria, J. \\ Department of Design and Manufacturing Engineering, University of Zaragoza, Spain \\ E-Mail: saguadoj@unizar.es, jesusve@unizar.es, dsamper@unizar.es,jsmazo@unizar.es
}

\begin{abstract}
Nowadays, the accuracy of machining parts is essential in order to compete in a global market as demanding as machining. Due to the high cost of these machines continuous operation is required, with a reduction of the downtime owing to production readiness, maintenance or breakdowns. Among all the sources of error that affect the accuracy of machining parts, this paper is focused on improving the positioning accuracy of the machine. This requires periodic inspection of the machine tool, which should be included in maintenance operations.

Volumetric verification is a novel technique that is being progressively introduced in machine tool maintenance operations, significantly reducing the time required. Unlike other techniques, this is based on non-linear mathematical models and the optimization process increases the influence factors. This modelling provides a re-creation of the whole verification process for all the influence factors; this allows the determination of the best measurement system distribution and the identification of techniques to use to characterize geometric errors. Thus, the verification time required is reduced, unnecessary tests are eliminated, the conditions under which real tests should be carried out are obtained in advance and machine tool accuracy is improved.

(Received in November 2015, accepted in March 2016. This paper was with the authors 1 week for 1 revision.)
\end{abstract}

Key Words: Laser Interferometry, Volumetric Verification, Simulation, Influence Factors, Accuracy, Machine Tool

\section{INTRODUCTION}

Currently a trend towards single parts with greater added value and accuracy is in demand on industrial markets such as in the rail, naval or wind sectors. Therefore machining flexibility and accuracy are required. To obtain both characteristics, machining systems require high positioning repeatability and a low influence of errors. Nowadays, with good enough repeatability of high performance machines, efforts are being focused on improving their accuracy.

A machine tool's (MT) performance and accuracy are affected by errors of its components and geometry. These can be divided into systematic errors and random errors. While systematic errors can be measured and modelled to reduce their influence using compensation methods, random errors are measured in order to avoid their influence but without compensating them [1-3]. The sources of errors can be classified in relation to their origin as dynamic effects [4], static load and motion control effects [5], thermal effects [6, 7] and kinematic/geometric errors [8-10]. The combination of all these sources of error provides the machining accuracy of the machine tool.

To improve the accuracy of the machine tool, the influence of its geometric errors can be reduced in two different ways. The first and more widespread one is based on direct measurement of each of the errors in the machine tool [11-13]. The second way to measure geometric errors is based on their indirect measurement. Indirect methods measure superposed errors associated with the simultaneous motion of two or more machine axes using systems such as a ball bar, laser tracer or laser tracker [14-16]. These ones require only one montage to measure the influence of all errors of the MT, reducing substantially the time required [10]. 
The principal problem of MT verification based on indirect measurement is the large number of factors that affect to it. Till today different researchers have focused on some of these one independently [17] or only analysing only an approach [18]. However, developed software allows us to face this problem from a global approach; merging scientific and industrial application in a simulation tool as in other areas is done [19,20]. Beyond study the influence of different factors previously unstudied, this paper presents a model which provides a re-creation of the whole verification process, addressing all the influence factors with these measurement systems. This makes it possible to determine the best measurement system distribution and to identify the techniques to use in order to characterize the geometric errors of the machine tool, improving its positioning accuracy after compensation. In this way, the verification time required is reduced, unnecessary tests are eliminated and the conditions under which real tests should be carried out are obtained in advance, reducing the time and improving the results.

\section{PROBLEM UNDER STUDY}

The main advantage of volumetric verification, compared to other verification techniques such as geometric verification based on direct measurement of errors, is the reduction of verification effort achieved [10]. However, the main disadvantage of volumetric verification is related with the process that is carried out, because the verification results will depend on test design pre-measuring on the machine.

As volumetric verification is based on indirect measurement of all errors simultaneously, it is necessary to obtain the mathematical relationship between them all. This depends on the structural components that define the sequence of movement of the machine tool (MT). The structure of a machine is determined by elements such as guides, joints and screws. This kinematic structure is modelled by a kinematic chain, which symbolises the flow of movements of serial kinematic structures. Geometric errors of linear and rotary axis are defined in ISO 230 and UNE 15300-1.

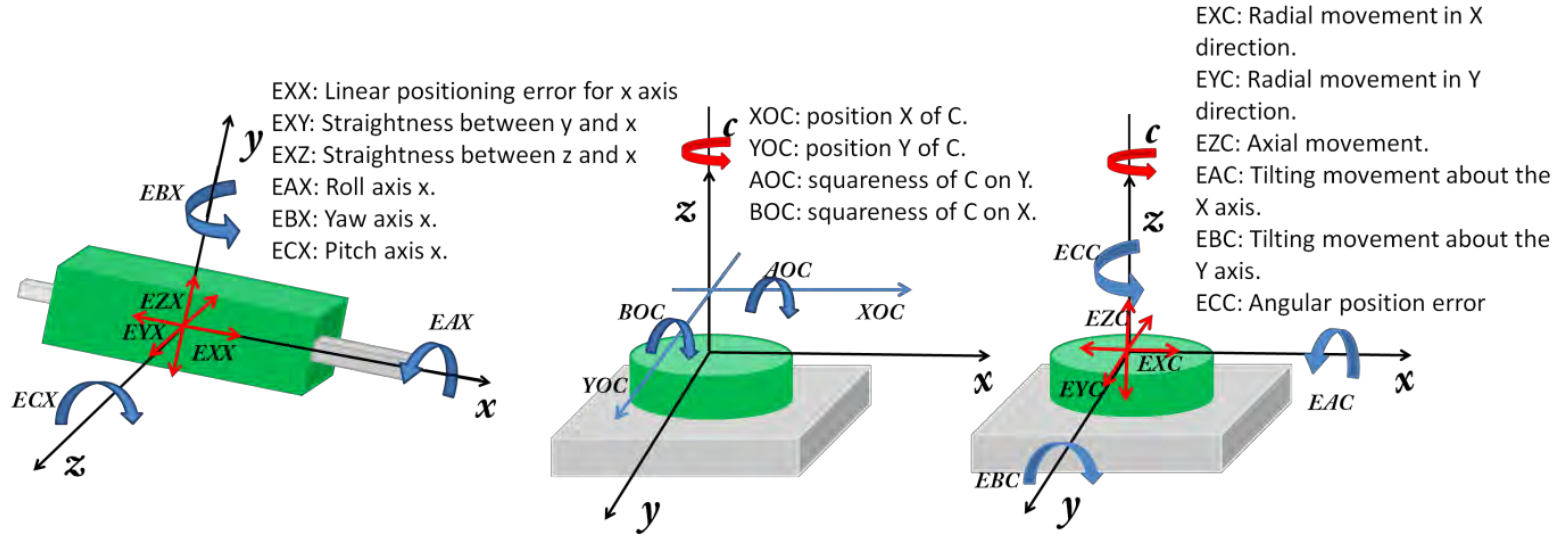

Figure 1: Geometric errors of linear X-axis \& geometric errors of a rotary C-axis.

Therefore, if a MT with three linear axes is verified, the number of errors present is six for each axis plus 3 squareness errors, yielding a total of 21 errors independently in the MT configuration. However, the relationship between geometric errors is different. If three MTs with different configurations FXYZ, XFYZ, and ZYXF are verified, the equation of movement that provides the relationship between all the geometric errors is different for each machine. $F$ determines the fixed part of the machine, Eqs. (1) to (3). The letters to the right of $F$ represent the axes that move with the tool, and letters to the left of $F$ represent the axes that move with the piece.

$$
\overline{\boldsymbol{R}(\boldsymbol{x})}\left(\overline{\boldsymbol{R}(\boldsymbol{y})}\left(\overline{\boldsymbol{R}(\boldsymbol{z})} \overline{\boldsymbol{T}}_{\boldsymbol{l}}+\overline{\boldsymbol{X}}\right)+\overline{\boldsymbol{Y}}\right)+\overline{\boldsymbol{z}}=\overline{\boldsymbol{T}_{\boldsymbol{t}}}
$$


where:

$$
\begin{gathered}
\overline{R(y)}\left(\overline{R(z)} \overline{T_{t}}+\bar{Z}\right)+\bar{y}=\overline{R(x)} \overline{T_{p}}+\bar{X} \\
\overline{T_{t}}=\overline{R(z)}\left(\overline{R(y)}\left(\overline{R(z)} \bar{T}_{p}+\bar{X}\right)+\bar{Y}\right)+\bar{z}
\end{gathered}
$$

$$
\begin{aligned}
& \overline{\boldsymbol{T}_{p}}=\left[\begin{array}{l}
x_{\boldsymbol{r}} \\
y_{r} \\
z_{r}
\end{array}\right] \text { represents the real coordinates of the MT when the part is machined. } \\
& \overline{\boldsymbol{T}_{T}}=\left[\begin{array}{l}
x_{t} \\
y_{t} \\
z_{t}
\end{array}\right] \text { represents the offset of the tool related with the machine head. }
\end{aligned}
$$

The position and straightness errors of the $\mathrm{x}, \mathrm{y}$ and $\mathrm{z}$ axis when the MT moves to $x_{n}, y_{n}, z_{n}$

$$
\bar{X}=\left[\begin{array}{c} 
\pm x_{n}+E X X \\
E Y X \\
E Z X
\end{array}\right] \bar{Y}=\left[\begin{array}{c}
E X Y \\
\pm y_{n}+E Y Y \\
E Z Y
\end{array}\right] \bar{Z}=\left[\begin{array}{c}
E X Z \\
E Y Z \\
\pm z_{n}+E Z Z
\end{array}\right]
$$

The sign of the displacement of the axis is positive if the axis moves the tool and negative if the axis moves the part. In addition, squareness error must be added to the $\overline{\boldsymbol{X}}, \overline{\boldsymbol{Y}}$ and $\overline{\boldsymbol{Z}}$ vectors that accumulate from the first axis to the workpiece or to the tool. For instance, in the MT with configuration $Z Y X F$ and three axis of movement, the linear errors are presented in Eqs. (9) to (11), where $S i j$ is the squareness error between axes $i$ and $j$.

$$
\bar{X}=\left[\begin{array}{c}
-x_{n}+E X X \\
-x_{n} S Y X+E Y X \\
-x_{n} S Z X+E Z X
\end{array}\right] \bar{Y}=\left[\begin{array}{c}
E X Y \\
-y_{n}+E Y Y \\
-y_{n} S Z Y+E Z Y
\end{array}\right] \bar{Z}=\left[\begin{array}{c}
E X Z \\
E Y Z \\
\pm z_{n}+E Z Z
\end{array}\right]
$$

$\overline{R(k)}$ - rotation error matrix on the $\mathrm{k}$ axis of the machine tool with $k=x, y, z$.

$$
\overline{R(k)}=\left(\begin{array}{ccc}
1 & E C k & -E B k \\
-E C k & 1 & E A k \\
E B k & -E A k & 1
\end{array}\right)
$$

The equation of movement of the MT relates nominal coordinates $P_{n}=\left(x_{n}, y_{n}, z_{n}\right)$ with geometric errors of the MT, providing the real position of the tool $P_{r}=\left(x_{r}, y_{r}, z_{r}\right)$. Volumetric verification seeks to reduce the difference between $P_{r}$ and $P_{n}$ in the whole machine workspace.

The measurement system used provides measured point $P_{m}=\left(x_{m}, y_{m}, z_{m}\right)$ related to nominal point $P_{n}$. Ideally, $P_{m}=P_{r}$; however, the influence of other sources of error such as measurement uncertainty, thermal variations, non-geometric errors of the MT, etc. is measured during data capture affecting the measured points $P_{m}$.

The set of nominal points to measure $P_{n}$ is defined as verification points $P_{v}$. The difference between the verification points and measured points is defined for us as the volumetric error of the machine ve, Eq. (13).

$$
v e=\sum_{i=1}^{n} \frac{\sqrt{\left(x_{m, i}-x_{n, i}\right)^{2}+\left(y_{m, i}-y_{n, i}\right)^{2}+\left(z_{m, i}-z_{n, i}\right)^{2}}}{n}
$$

Volumetric verification reduces the volumetric error of the machine using non-linear optimization techniques to characterize the joint influence of all geometric errors through the equation of movement of the MT. Nevertheless, the laser tracker (LT) provides coordinates of the measured points in its own coordinate system (CSLT), while the nominal points are in the machine tool's coordinate system (CSMT). So, the LT must be introduced in the kinematic chain in the location of the part. If the coordinates of the LT provided by the equations of movement (Eqs. (14) to (16) related to Eqs. (1) to (3)) are compared with the real coordinates measured by the LT, the error to minimize is obtained.

$$
\overline{\mathbf{X}_{\mathbf{L T}}}=\overline{\mathbf{R}_{\mathbf{L T}}^{-1}}\left(\overline{\mathbf{X}}+\overline{\mathbf{R}(\mathbf{x})^{-1}}\left(\overline{\mathbf{Y}}+\overline{\mathbf{R}(\mathbf{y})^{-1}}\left(\overline{\mathbf{Z}}+\overline{\mathbf{R}(\mathbf{z})^{-1}} \overline{\mathbf{T}_{\mathbf{T}}}\right)\right)-\overline{\mathbf{T}_{\mathbf{L T}}}\right)
$$




$$
\begin{gathered}
\overline{\mathbf{X}_{\mathbf{L T}}}=\overline{\mathbf{R}_{\mathbf{L T}}^{-1}}\left(\overline{\mathbf{R}(\mathbf{x})}\left(\overline{\mathbf{Y}}+\overline{\mathbf{R}(\mathbf{y})^{-1}}\left(\overline{\mathbf{Z}}+\overline{\mathbf{R}(\mathbf{z})^{-1}} \overline{\mathbf{T}_{\mathbf{T}}}\right)-\overline{\mathbf{X}}\right)-\overline{\mathbf{T}_{\mathbf{L T}}}\right) \\
\overline{\mathbf{X}_{\mathbf{L T}}}=\overline{\mathbf{R}_{\mathbf{L T}}^{-1}}\left(\overline{\mathbf{R}(\mathbf{x})}(\overline{\mathbf{R}(\mathbf{y})}(\overline{\mathbf{R}(\mathbf{z})}(\overline{\mathbf{T}}-\overline{\mathbf{Z}})-\overline{\mathbf{Y}})-\overline{\mathbf{X}})-\overline{\mathbf{T}_{\mathbf{L T}}}\right) \\
v e=\sum_{i=\mathbf{1}}^{n} \frac{\sqrt{\left(\mathbf{x}_{\mathrm{LTm}, \mathrm{i}}-\mathbf{x}_{\mathrm{LT}, \mathrm{i}}\right)^{2}+\left(\mathbf{y}_{\mathrm{LTm}, \mathrm{i}}-\mathbf{y}_{\mathrm{LT}, \mathrm{i}}\right)^{2}+\left(\mathbf{z}_{\mathrm{LTm}, 1}-\mathbf{z}_{\mathrm{LT}, \mathrm{i}}\right)^{2}}}{n}
\end{gathered}
$$

As shown in the equations presented above, the volumetric error depends on:

- The structural configuration of the MT to be verified.

- The number and spatial distribution of the verification points.

- The characteristics of the measurement system used.

- The spatial location of the measurement systems around the workspace of the MT.

Once the initial volumetric error is obtained, non-linear optimization techniques are used to reduce the geometric errors of the MT, obtaining the approximation functions for each error. Nominal points introduced in the kinematic model of the machine are influenced by the geometric errors of the machine, modifying the points' coordinates. As the error values are not known, regression functions that provide the values of each error for each coordinate of each point are used as the geometric errors of the kinematic model. In order to reduce the volumetric error of the machine, the values of the coefficients that define the regression functions change during the optimization process. When the optimization process is over, the approximation functions obtained are used to compensate their joint influence (Fig. 2).

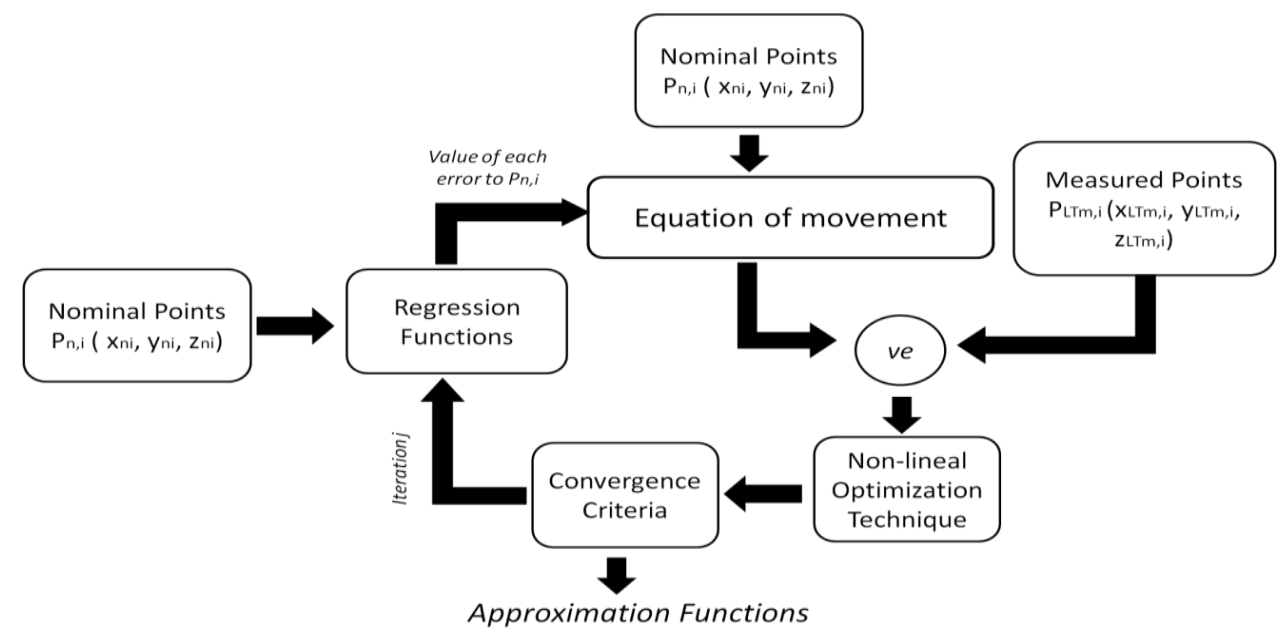

Figure 2: Scheme of volumetric verification process.

\section{INFLUENCES ON MT VOLUMETRIC VERIFICATION}

To tackle the volumetric verification, the sources of errors have been divided according to the part of the process where they are introduced (Fig. 3):

- Errors of the machine tool. Besides geometric errors, the MT is affected by errors such as backlash errors, repeatability of the machine, tracking and control errors. All of these are related with the spatial distribution of the measurement points used for verification (verification points). Therefore, different numbers of verification points, the distribution of points and the direction from which the MT moves all affect the verification.

- Errors of the measurement system. The use of a measurement system based on laser interferometry is affected by environmental conditions of pressure, temperature and humidity [21]. Although these influences are reduced, uncertainty of the angular encoders and interferometer, the measurement condition and user influences are random errors which cannot be compensated but can be reduced. To do so, good laser tracker positioning and a correct distribution of verification points is required. 
- Errors of measurement strategy. Once the identification process begins, the most important source of error is uncertainty introduced by the roto-translation matrix $\overline{\boldsymbol{R}_{L T}}$ which relates CSLT and CSMT. If measurement is carried out using an LT in a position, only a value of $\overline{\boldsymbol{R}_{\boldsymbol{L T}}}$ is needed. However, if the same LT is located at three different positions to apply multilateration techniques [22], four different values of $\overline{\boldsymbol{R}_{L T, \boldsymbol{l}=1 . . n}}$ are required, thus increasing the positioning uncertainty introduced.

- Errors of identification strategy. To obtain approximation functions the identification process can be constituted with a different optimization process according to the strategy defined by the user [22].

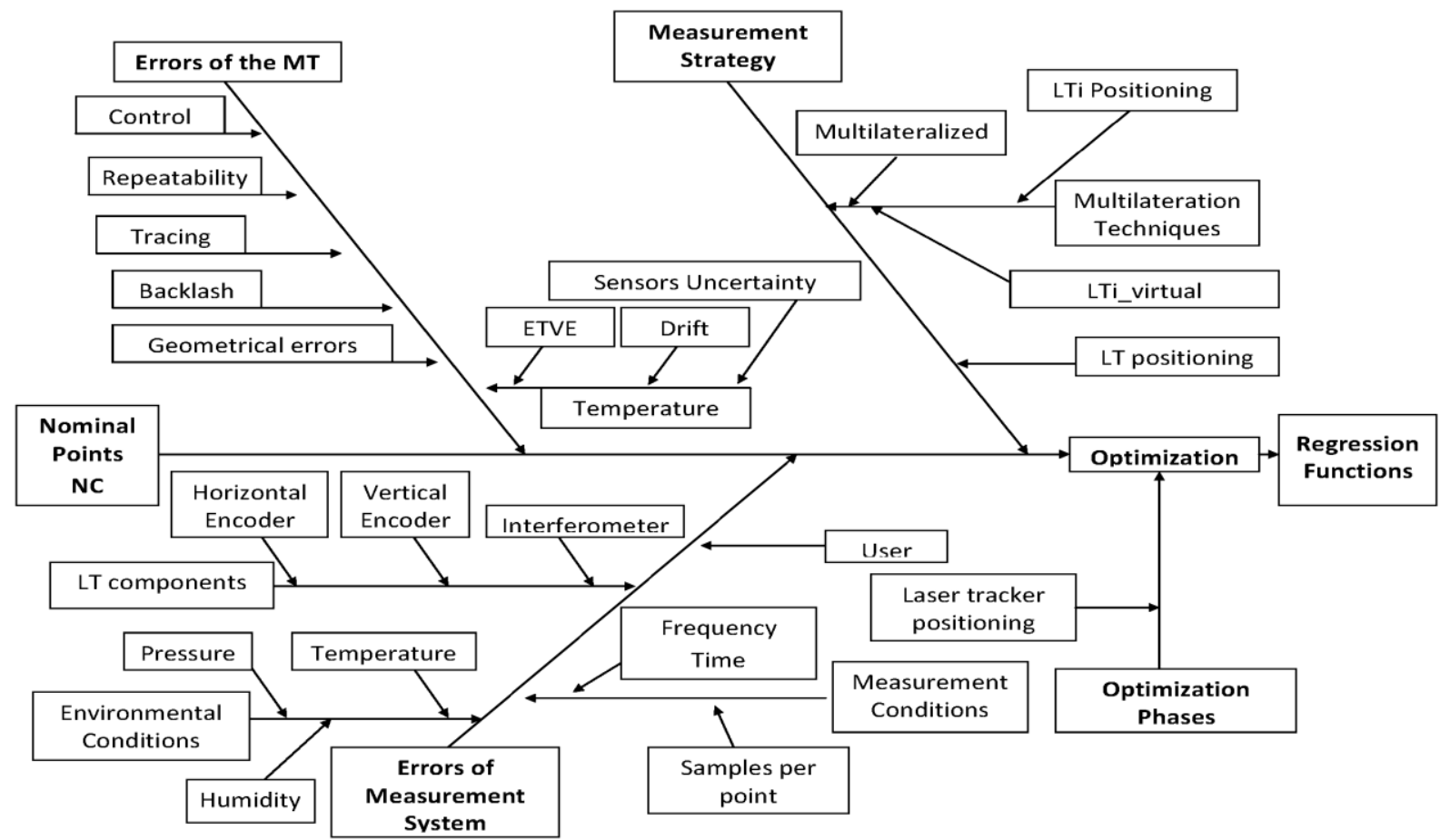

Figure 3: Influence of error in volumetric verification based on LT measurements.

All the influences of errors are divided into sources of errors in which the test definition can predict their behaviour, and sources of error which influence the MT accuracy should be measured and determined in-situ. To estimate their uncertainty Monte Carlo technique is required [23].

\section{MODEL AND IMPLEMENTATION}

The computer-assisted verification process developed involves five different steps. Both the main applications developed as independent functions created for this computer assistant have been scheduled using the commercial application Matlab.

\subsection{Determination of the kinematic model and equation of movement of the MT}

This consists of a set of algorithms that allow the user of the application to obtain the kinematic model of any machine, from three to $n$ axes, and the equation of motion, including the measurement system (Fig. 4). The main inputs of the software are:

- Number of axes of the machine tool.

- Sequence of movements of the kinematic chain, from which it obtains the position of the workpiece. This indicates the different axes of movement that are involved and in which order. 
- Sequence of movements from which it obtains the position of the tool, indicating the axes of movement that are involved and in which order.

All this information can be introduced by the user using a database where the more frequent structural configurations can be found, or using an editable area.

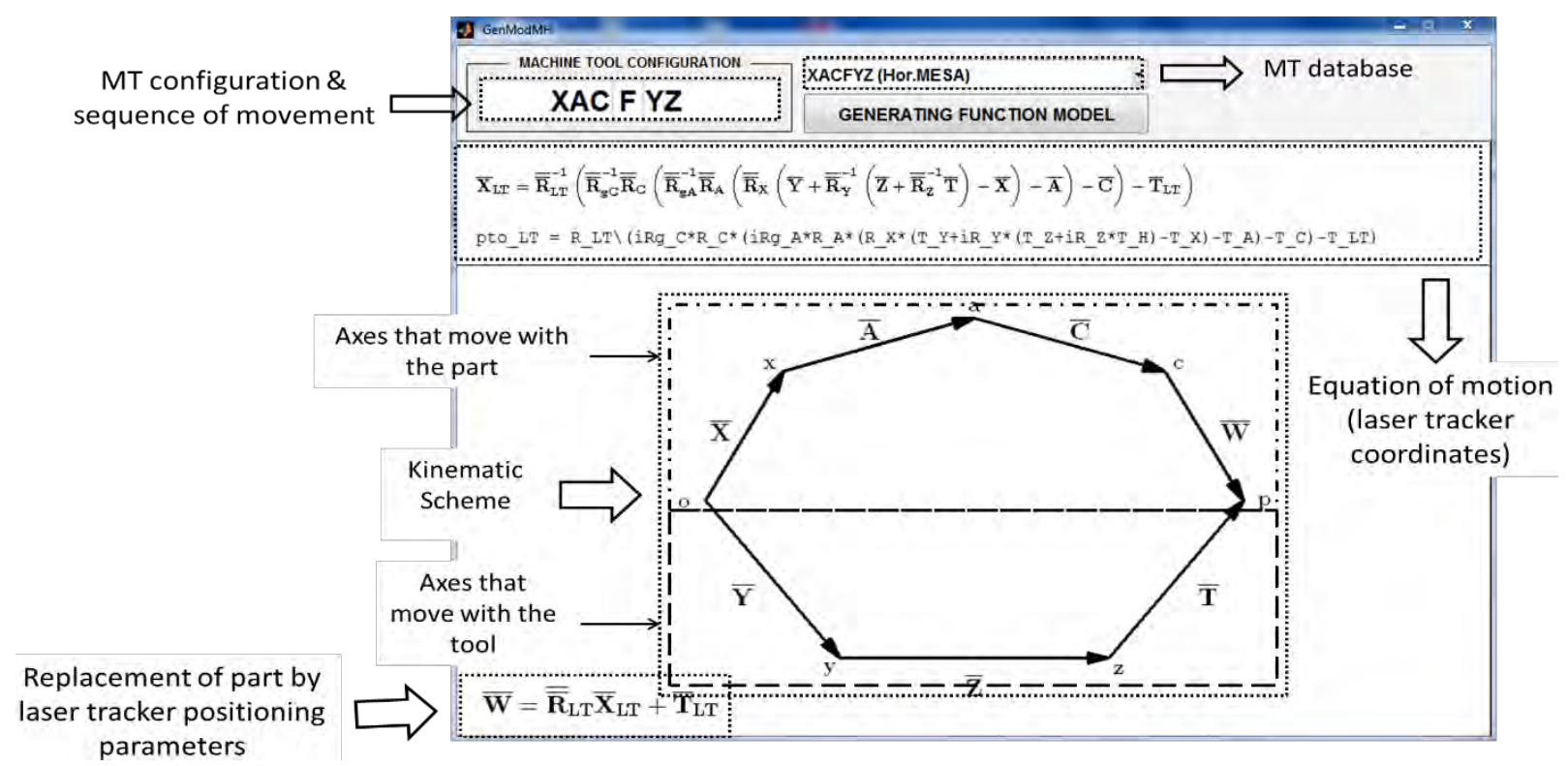

Figure 4: Machine tool kinematic model generator.

The outputs provided by the generator are:

- A graphical representation of the kinematic scheme of the machine tool.

- The equation of motion of the machine. This shows the coordinates measured by the laser tracker through the geometric machine errors, machine offset and nominal coordinates entered.

- An enterable file from the volumetric verification software that contains both the function of the kinematic model of the machine and the information necessary for its use.

\subsection{Discretization of MT workspace}

The set of algorithms then discretizes the workspace of the machine to different types of mesh and paths [24] depending on the error to be characterized. The main inputs of the software are:

- Structural configuration of the machine.

- Initial coordinates of each of the axes of the machine.

- Maximum value of the coordinates of each of the axes of the machine.

- Step between two consecutive points in each of the axes.

- Motion sequence of the axes. $X A N D Z$

- Overshoot of each axis. [50 25 15]

- Grid type discretization (direction of the axes).

- Filename with the head of the CNC program.

- Filename format with the CNC program.

- CNC program file name to be generated.

- Extension of the CNC program.

- Discretization shaped mesh (0), cloud (1), diagonals (2).

- Letter used by the control to determine downtime.

- Stop time at each point in seconds. 
According to the areas of interest of the MT, the verification points to measure can be distributed as a mesh or cloud of points. Mesh workspace discretization presents redundant information. Alternatively, a cloud of points with the same number of points provides more information on intermediate points than using a mesh of points.

To obtain the cloud of points while maintaining the direction of movement of the axes, the software generates a uniform mesh of points. Subsequently, each point's coordinates are affected by a random value as in Eq. (18). Similarly, verification points can be distributed using diagonals of the MT workspace, thus reducing the measured points, but losing information inside the MT.

$$
\left.x_{\text {cloud }}=x_{\text {mesh }} \pm \text { rand (interval }(x)\right) \text { if } x>x_{\text {min }} \text { and } x<x_{\text {max }}
$$

One of the most relevant sources of error is the backlash errors of the MT. Thus, the influence of the direction of movement, backlash errors, the number of points and their spatial distribution in the volumetric verification can be modelled and studied.

\subsection{Introduction of geometric errors in mesh of points}

The nominal verification points created during discretization must be affected by geometric errors to re-create the real behaviour of the machine.

The developed software allows the user to introduce the influence of each error individually. Each error is introduced in the model using a generation function throughout its domain. As the behaviour of the geometric errors generated depends on the generation functions used, these can be introduced as simple, Legendre or Chebyshev polynomials when errors of linear axes are introduced, and as a Fourier series for errors from rotary axes (Fig. 5).

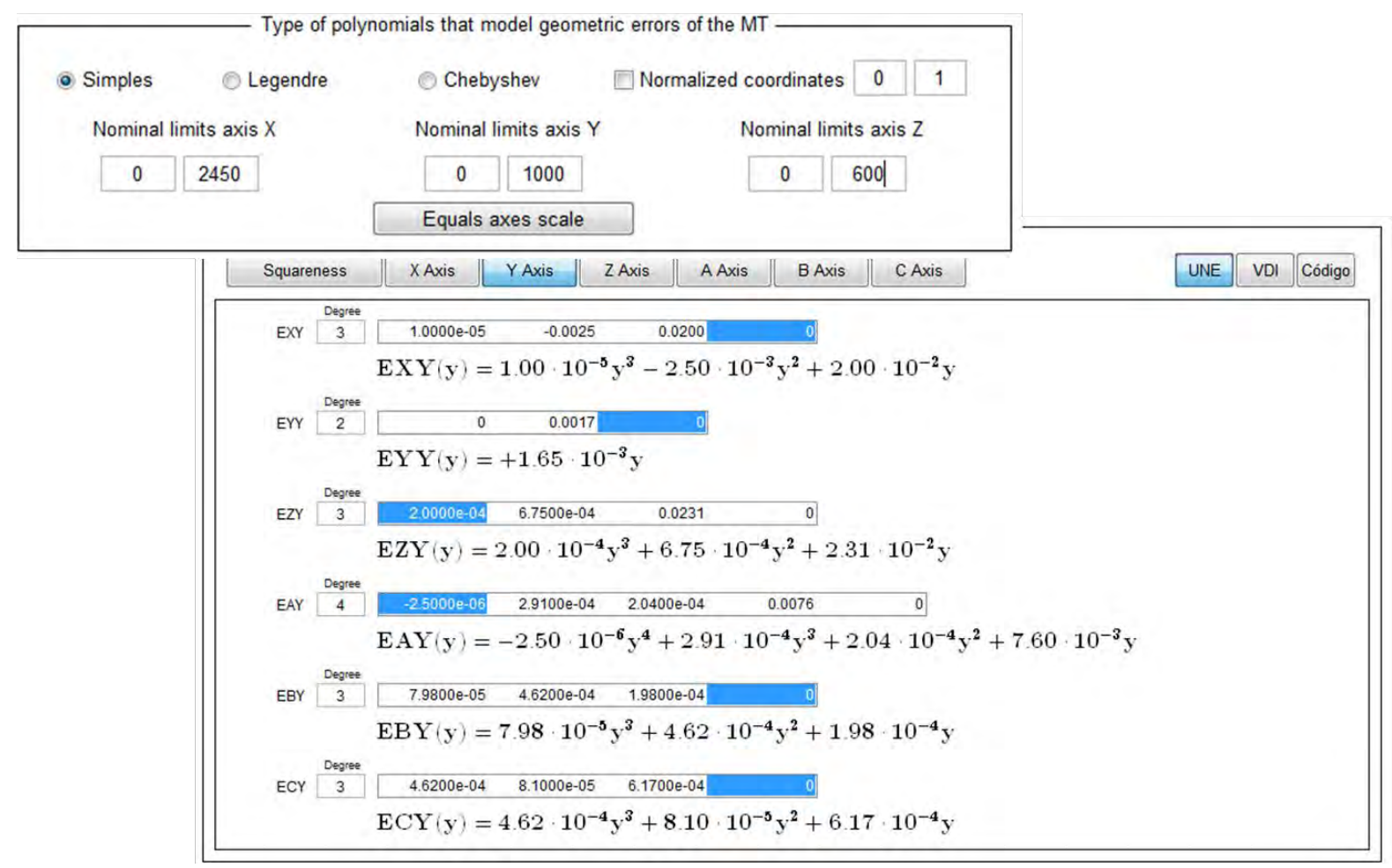

Figure 5: Introduction of geometric errors of the MT into simulation model.

For each nominal point the value of each geometric error is evaluated using a generation function. Then the nominal coordinates and values of each error are introduced into the equation of movement obtained through the kinematic model of the MT. It then provides the coordinates of the nominal point affected by the combined effect of all geometric errors. 


\subsection{Number of laser trackers and positioning}

A LT provides the position of a point in spherical coordinates. This position is determined by comparing a measurement beam and a reference beam from the combination of a laser interferometer and the readings of azimuth and polar coordinates of the angular encoders. LTs from different manufacturers have different measurement characteristic and origins.

The positioning of LTs has been modelled in two different ways. The first is based on a rotation matrix and a translation vector that provide the position of the LT as a least squares adjustment. These may be entered manually by the user, or automatically through previous tests generated. The second is based on Euler angles and the translation vector (Fig. 6).

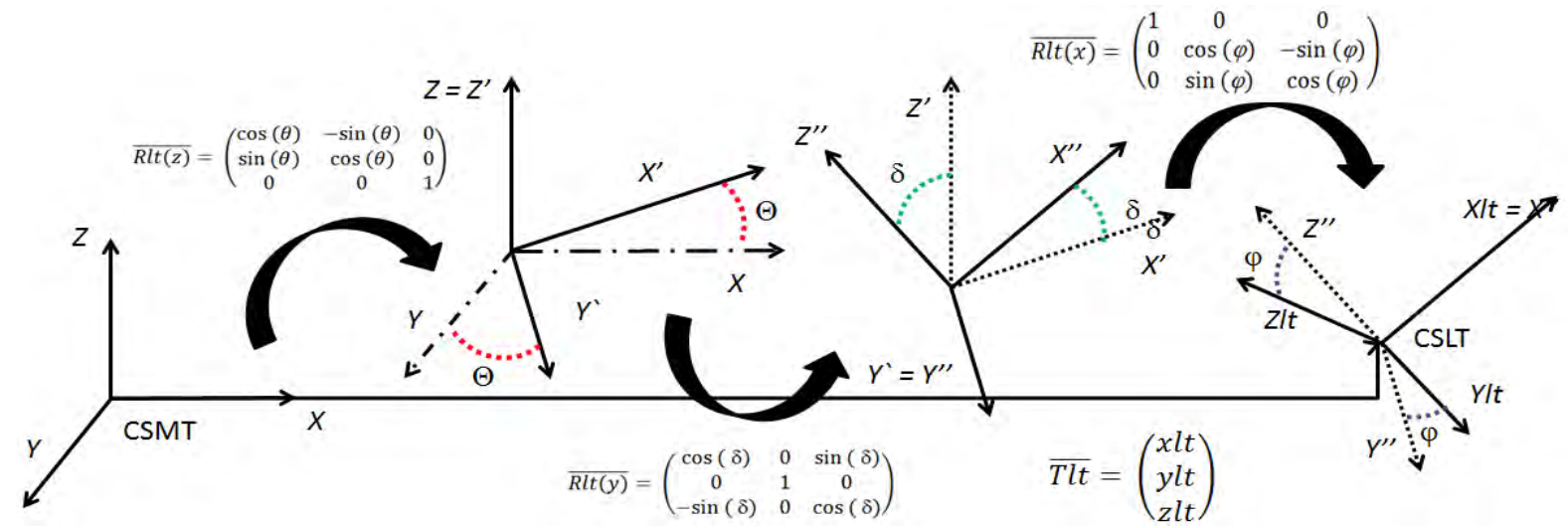

Figure 6: Relationship between CSMT \& CSLT using Euler angles.

The position of the measurement system defined by $\overline{\boldsymbol{R l t}}$ and $\overline{\boldsymbol{T l t}}$ should not be generated randomly. The development of this simulation model allows the following to be taken into consideration:

- Workspace to be verified. Depending on the structure of the machine tool, fairing, areas of interest, etc.

- Azimuth and elevation tracking angle of retro-reflector to use.

- Azimuth and elevation tracking angle of laser tracker used.

- Minimum and maximum height of the laser tracker using extenders.

The model checks if the LT position satisfies vision restriction as shown in Fig. 7.

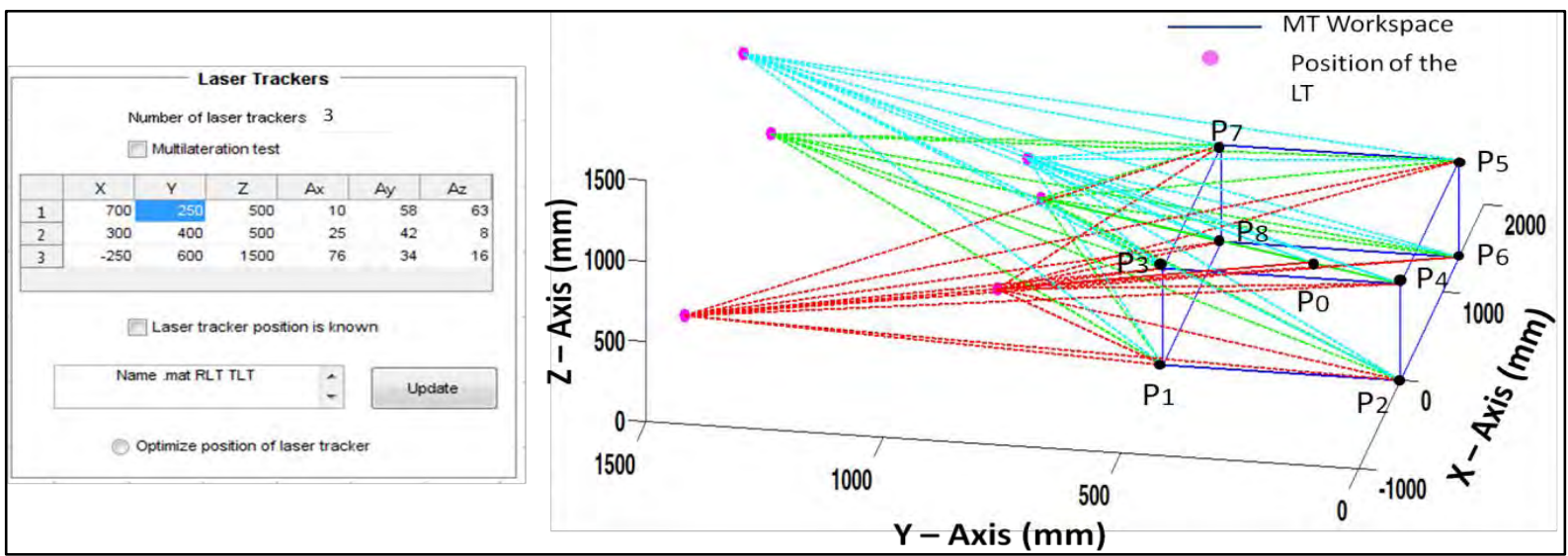

Figure 7: Spatial distribution of laser tracker.

The model limits the MT workspace using nine points. The first $\left(\mathrm{P}_{0}\right)$ is located in the middle of the workspace. Points from $\mathrm{P}_{1}$ to $\mathrm{P}_{9}$ are defined by the upper and lower limits of each axis of movement. 
From the LT position the model generates the measurement beam between the LT and retro-reflector $\left(r_{L T, 0 . .} r_{L T, 9}\right)$. At $\mathrm{P}_{0}$ the measuring beam should be incident at the retro-reflector with an angle of $0^{\circ}$ approximately. Therefore, it is taken as the reference. If the spatial angle between $\overline{\boldsymbol{r}_{\boldsymbol{L T}, \mathbf{0}}}$ and $\overline{\boldsymbol{r}_{\boldsymbol{L T}, \boldsymbol{l}}}$ with $i=1 \ldots n$ is lower than the maximum and greater than the minimum vision angle of the retro-reflector (Eqs. (19) to (21)), the LT sees it on the whole MT workspace.

$$
\begin{gathered}
\bar{u}=\overline{r_{L T, 0}}=\left(x_{p 0}-x_{L T 0}, y_{p 0}-y_{L T 0}, z_{p 0}-z_{L T 0}\right) \text { with } x_{L T 0}=y_{L T 0}=z_{L T 0}=0 \\
\bar{v}=\overline{r_{L T, \imath}}=\left(x_{p i}-x_{L T 0}, y_{p i}-y_{L T 0}, z_{p i}-z_{L T 0}\right) \\
\alpha_{\text {min }} \leq \alpha_{i} \leq \alpha_{\text {max }} \text { with } \alpha_{i}=\operatorname{acos}\left(\frac{u_{1} v_{1}+u_{2} v_{2}+u_{3} v_{3}}{\sqrt{u_{1}^{2}+u_{2}^{2}+u_{3}^{2}} \sqrt{v_{1}^{2}+v_{2}^{2}+v_{3}^{2}}}\right)
\end{gathered}
$$

The model also allows the user to introduce three and/or four laser trackers. Therefore, it is able to re-create the measurement of the same point from three or four positions, obtaining new coordinates using multilateration and auto-calibration techniques [25].

\subsection{Introduction of influences of measurement uncertainty}

Greater contributions to the measurement uncertainty [26-27] are the uncertainty of the interferometer and the uncertainty of the angular encoders. Uncertainties of the radial and angular coordinates are modelled as a normal distribution. Radial uncertainty is modelled with a constant term plus another dependent on the measurement distance, Eq. (22). Meanwhile angular uncertainties are modelled as constant terms, Eqs. (23) and (24).

$$
\begin{gathered}
\boldsymbol{u}_{\boldsymbol{r}}=\boldsymbol{a}(\mu \boldsymbol{m})+\boldsymbol{b}(\mu \boldsymbol{m}) / \boldsymbol{m} \\
\boldsymbol{u}_{\alpha}=\boldsymbol{c}(\boldsymbol{r a d}) \\
\boldsymbol{u}_{\boldsymbol{\beta}}=\boldsymbol{d}(\boldsymbol{r a d})
\end{gathered}
$$

When the simulation model provides the coordinates that re-create the measurement of the laser tracker $\overline{X_{L T}}$, these are transformed into spherical coordinates and are affected by uncertainty of the measurement system. When this transformation is undone, the Cartesian coordinates are affected by the measurement uncertainty.

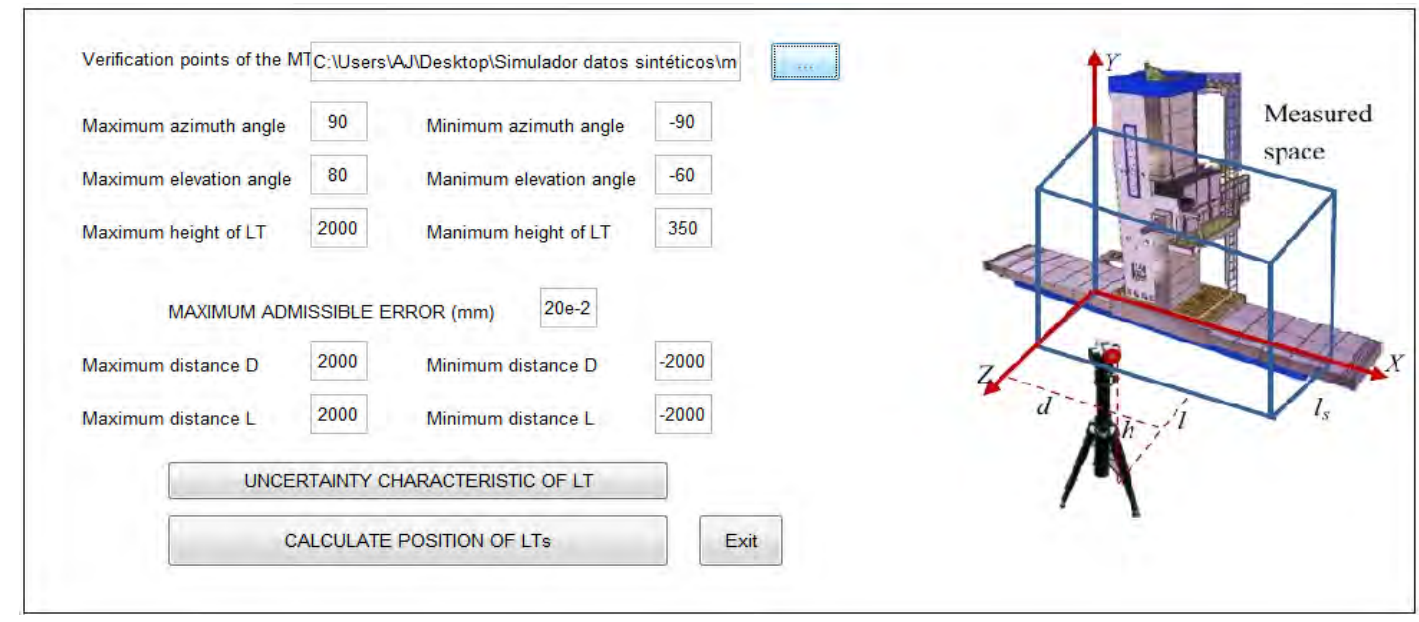

Figure 8: Conditions of the laser tracker positioning.

The simulation model includes a tool from which the position of the LT can be optimized in order to reduce its influence on the measurement uncertainty. This takes into consideration the maximum and minimum values of the azimuth and elevation angles, the maximum and minimum height of the LT, the distribution of verification points, the maximum admissible error and the uncertainty characteristics of the LT (Fig. 8). 


\section{TEST CARRIED OUT}

To show the usefulness of the simulation model, the verification of an MT with configuration XFYZ has been re-created with different measurement conditions. The geometric errors of the MT, the workspace to verify and the identification strategy are constant, but the LT positioning, number of verification points and distribution change.

The machine tool workspace was defined as $0 \mathrm{~mm} \leq x \leq 2500 \mathrm{~mm}, 0 \mathrm{~mm} \leq y \leq 1500 \mathrm{~mm}$ and $0 \mathrm{~mm} \leq \mathrm{z} \leq 1200 \mathrm{~mm}$ with intervals of $500 \mathrm{~mm}$ in the $x$ axis, $500 \mathrm{~mm}$ in the $y$ axis and $400 \mathrm{~mm}$ in the $z$ axis, creating a verification mesh of 96 points.

First of all, the influence of the position of the laser tracker is studied. Table I shows the positions of the laser tracker. The first three positions are determined manually, while the last one is obtained using the tool presented in section 4.5 to reduce the influence of measurement uncertainty in the verification process. First a series of tests to identify MT geometric errors but without the influence of measurement uncertainty were created. The verification results in Table 1 show the initial and final volumetric error of the MT. If measurement uncertainty is not considered, the results are the same for all LT positions.

Table I: Positions of LT and verification results without measurement uncertainty.

\begin{tabular}{|c|c|c|c|c|c|c|}
\hline \multicolumn{7}{|c|}{ Laser Tracker Position } \\
\hline $\mathbf{L T}$ & $\mathbf{X}(\mathrm{mm})$ & $\mathbf{Y}(\mathrm{mm})$ & $\mathbf{Z}(\mathrm{mm})$ & $\alpha^{\circ}$ & $\beta^{\circ}$ & $\varphi^{\circ}$ \\
\hline Pos 1 & 3500 & -1500 & 500 & 15 & 37 & 26 \\
\hline Pos 2 & 4500 & -800 & 500 & 32 & 17 & 12 \\
\hline Pos 3 & 1250 & 2000 & 600 & 5 & 65 & 32 \\
\hline Pos 4 & 1100 & -1000 & 600 & 63 & 14 & 35 \\
\hline & \multicolumn{3}{|c|}{ Initial Volumetric Error $(\mu \mathrm{m})$} & \multicolumn{3}{|c|}{ Final Volumetric Error $(\mu \mathrm{m})$} \\
\hline LT & Maximum & Average & Minimum & Maximum & Average & Minimum \\
\hline Pos 1...4 & 63.7 & 35.3 & 0.0 & 16.3 & 7.7 & 0.0 \\
\hline
\end{tabular}

Afterwards the same tests were generated by adding the measurement noise of the LT. It was modelled as a normal distribution with [24 $\mu \mathrm{rad}, 24 \mu \mathrm{rad}, 4 \mu \mathrm{m}+0.8 \mu \mathrm{m} / \mathrm{m}$ ] to the angular and radial encoders. Table II shows the verification results using the same verification process.

Table II: Results of verification process - mesh of 96 points.

\begin{tabular}{|c|c|c|c|c|c|c|}
\hline & \multicolumn{3}{|c|}{ Initial Volumetric Error $(\mu \mathrm{m})$} & \multicolumn{3}{c|}{ Final Volumetric Error $(\mu \mathrm{m})$} \\
\hline LT & Maximum & Average & Minimum & Maximum & Average & Minimum \\
\hline Pos 1 & 121.1 & 72.0 & 44.8 & 81.4 & 41.6 & 7.7 \\
\hline Pos 2 & 85.0 & 53.1 & 29.4 & 55.3 & 25.9 & 1.7 \\
\hline Pos 3 & 122.1 & 71.2 & 43.0 & 74.3 & 32.3 & 2.6 \\
\hline Pos 4 & 111.1 & 70.0 & 26.8 & 40.8 & 21.5 & 4.4 \\
\hline
\end{tabular}

The results of Table II show that the volumetric error is strongly affected by the measurement uncertainty, thus increasing the initial and final volumetric error of the MT. This is related with the laser tracker positions, providing the least influence when the positioning tool is used.

The computer assistant developed also allows the user to analyse the influence of the distribution and number of verification points. To study the influence of the points' distribution, the same MT workspace was discretized as a cloud instead of a mesh of points, 
following Eq. (18). Table III shows the results of the verification process with the same number of verification points.

Table III: Results of verification process - cloud of 96 points.

\begin{tabular}{|c|c|c|c|c|c|c|}
\hline & \multicolumn{3}{|c|}{ Initial Volumetric Error $(\mu \mathrm{m})$} & \multicolumn{3}{c|}{ Final Volumetric Error $(\mu \mathrm{m})$} \\
\hline LT & Maximum & Average & Minimum & Maximum & Average & Minimum \\
\hline Pos 1 & 121.1 & 72.0 & 45.5 & 81.9 & 32.8 & 2.5 \\
\hline Pos 2 & 85.0 & 53.4 & 29.4 & 55.3 & 26.1 & 4.4 \\
\hline Pos 3 & 140.7 & 88.6 & 29.4 & 61.5 & 39.0 & 6.6 \\
\hline Pos 4 & 108.3 & 65.3 & 25.6 & 37.1 & 16.3 & 3.0 \\
\hline
\end{tabular}

Comparing Table II and Table III, it is observed that the initial volumetric error remains constant when the LT is located in position 1 and position 2. However, the LT at positions 3 and 4 provides a greater variation when a cloud of verification points is used. The verification results differ between the cloud and mesh of points. The LT at position 4 provides an improvement of approximately $24 \%$ when the verification points change from a mesh to a cloud of points.

Increasing the number of verification points provides more information on the MT accuracy but increases exponentially the verification time required. The MT workspace was defined as $0 \mathrm{~mm} \leq x \leq 2500 \mathrm{~mm}, 0 \mathrm{~mm} \leq y \leq 1500 \mathrm{~mm}$ and $0 \mathrm{~mm} \leq z \leq 1200 \mathrm{~mm}$ with an interval of $100 \mathrm{~mm}$ in the $x$ axis, $100 \mathrm{~mm}$ in the $y$ axis and $100 \mathrm{~mm}$ in the $z$ axis, creating a verification mesh of 5408 points (Fig. 9).

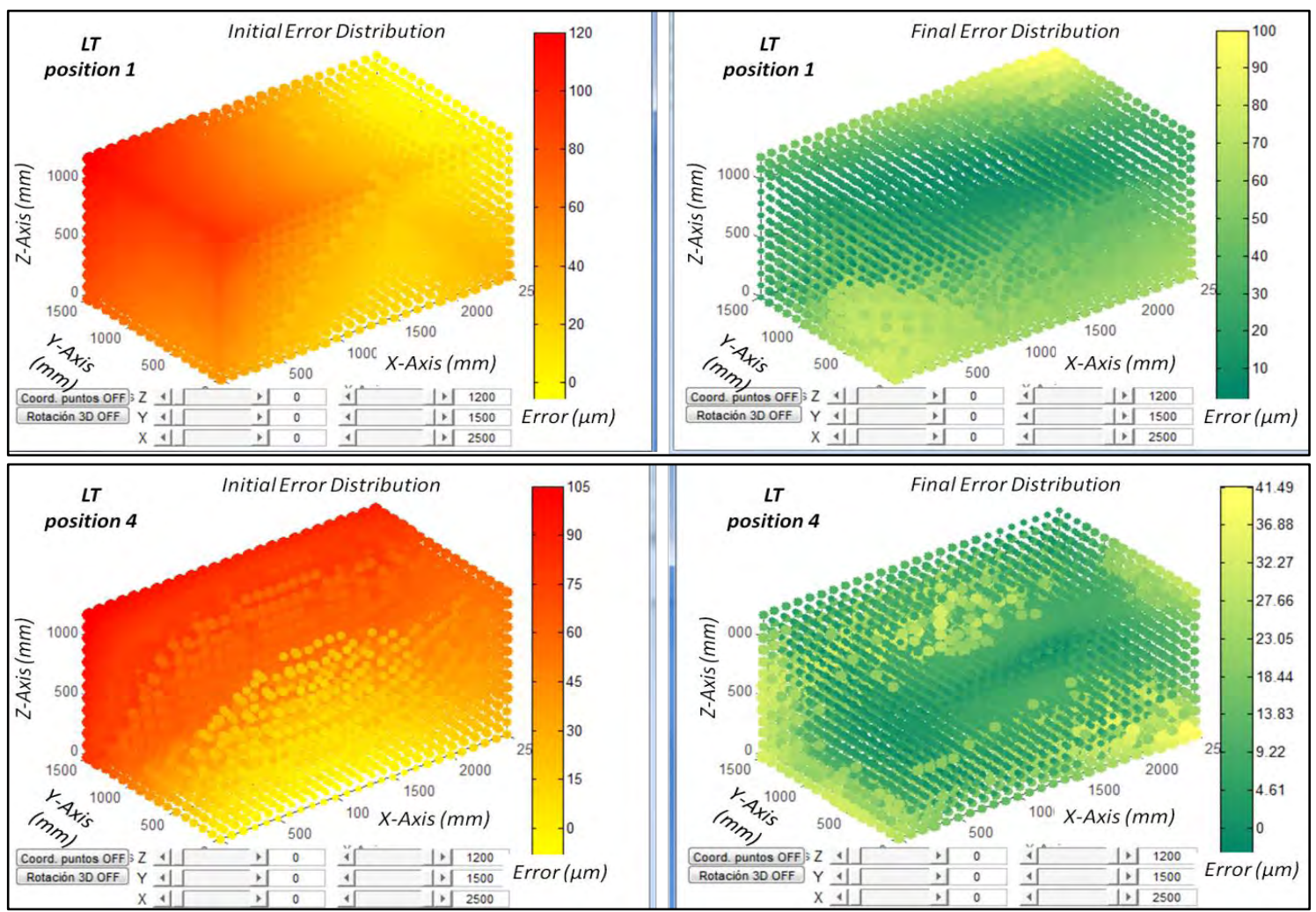

Figure 9: Initial and final error distribution with LT in positions $1 \& 4-$ mesh of 5408 points.

If the verification times of mesh 1 (96 points) and mesh 2 (5408 points) are compared, the preparation time is the same, but the measuring time increases from 25 minutes to approximately 9 hours (assuming a stabilisation time of 6 seconds per point). This difference 
is increased due to acceleration and deceleration between the measuring points. In the same way, the computational cost is increased from 15 minutes to 3.5 hours.

Table IV shows the results of the verification process. These show that the LT position that provides the best results is position 4, just as a result of the small mesh of points. Therefore, a large number of points is not required to obtain the best laser tracker position and can reduce the time required by the location optimization process.

Table IV: Results of verification process - mesh of 5408 points.

\begin{tabular}{|c|c|c|c|c|c|c|}
\hline & \multicolumn{2}{|c|}{ Initial Volumetric Error $(\mu \mathrm{m})$} & \multicolumn{3}{c|}{ Final Volumetric Error $(\mu \mathrm{m})$} \\
\hline LT & Maximum & Average & Minimum & Maximum & Average & Minimum \\
\hline No noise & 63.7 & 33.7 & 0.0 & 14.6 & 6.6 & 0.0 \\
\hline Pos 1 & 121.1 & 69.9 & 44.8 & 98.2 & 35.1 & 1.9 \\
\hline Pos 2 & 85.2 & 50.2 & 29.2 & 88.0 & 47.0 & 22.2 \\
\hline Pos 3 & 140.7 & 87.0 & 6.15 & 113.4 & 40.5 & 1.2 \\
\hline Pos 4 & 108.3 & 63.6 & 25.3 & 46.1 & 16.3 & 8.0 \\
\hline
\end{tabular}

Comparing Table I and Table IV shows that the volumetric error of the machine is increased by only $1.4 \mu \mathrm{m}$ when the number of points is increased and the measurement uncertainty is not considered. In the same way, the final error is increased by only $1.1 \mu \mathrm{m}$. Therefore, to characterize the influence of geometric errors a large number of verification points is not required if there is no measurement uncertainty.

When the measurement uncertainty is taken into consideration (Table II and Table IV), the behaviour of the volumetric error is different at each LT position. When the LT is located in position 1 and position 2, the initial volumetric error is similar. However, when the LT is located in positions 3 and 4 the initial error increases by approximately $22 \%$ and $35 \%$ respectively. This may be due to an increase of the influence of the measurement noise or a bad initial value of the volumetric error as a result of a bad workspace discretization. The results from both tables show that the final error changes too. However, the best laser tracker verification position is position 4 , obtained by the positioning tool too. The final volumetric error is reduced by $6 \mu \mathrm{m}$ more $(\approx 38 \%)$, but the verification time is increased at a far greater rate.

If the results from the cloud (Table III) and mesh of points (Table IV) are analysed, it is observed that both distributions yield similar results. But the verification and computational time required is much smaller when using a cloud instead of a mesh of points with a higher number of points.

\section{CONCLUSION}

The design phase is one of the most important steps in the volumetric verification process. It permits the user to determine the best verification options under which error sources have the least influence on the measurement of volumetric error. It provides more accurate approximation functions to improve the machine tool's accuracy. The methodology and tools presented in this paper offer a way to create and validate the "know-how" on volumetric verification, avoiding unnecessary tests and improving the results.

The computer assistant allows the user to re-create a real verification of any type of machine, depending on the machine tool configuration and measurement system used. The assistant enables the user to obtain the equation of motion of an MT within the laser tracker, create a real numerical control program with verification points with different point distributions, determine the position of the laser tracker to reduce the influence of 
measurement uncertainty, and model the influence of both the uncertainty of the laser tracker and machine tool errors. Tests carried out show that the verification results depend strongly on the modelled parameters, improving the results when a good test design is realized.

Our aim is that this paper should assist maintenance and quality control operators, helping them to tailor the design parameters to the measurement requirements. Also, this paper will enable the quality assurance process to become a key point in the design of the verification process, where different influence factors can be modelled and analysed.

\section{ACKNOWLEDGEMENT}

This work was supported by Spanish Government (Ministry of Economy and Competitiveness) through the research project METRAP - Development of new techniques of verification and compensation of manufacturing systems for traceable in process metrology (DPI2013-46979-C2-1-P), Scientific and Technical Research of Excellence Development National Program, and by Aragon Government (Department of Industry and Innovation) through the Research Activity Grant for research groups recognized by the Aragon Government (2014-T62 Manufacturing Engineering and Advanced Metrology Group).

\section{REFERENCES}

[1] Chaari, R.; Abdennadher, M.; Louati, J.; Haddar, M. (2011). Modelling of the 3D machining geometric defects accounting for workpiece vibratory behaviour, International Journal of Simulation Modelling, Vol. 10, No. 2, 66-77, doi:10.2507/IJSIMM10(2)2.173

[2] Ramesh, R.; Mannan, M. A.; Poo, A. N. (2000). Error compensation in machine tools - a review: Part I: geometric, cutting-force induced and fixture-dependent errors, International Journal of Machine Tools and Manufacture, Vol. 40, No. 9, 1235-1256, doi:10.1016/S08906955(00)00009-2

[3] Tamizharasan, T.; Senthil Kumar, N. (2012). Optimization of cutting insert geometry using DEFORM-3D: Numerical simulation and experimental validation, International Journal of Simulation Modelling, Vol. 11, No. 2, 65-76, doi:10.2507/IJSIMM11(2)1.200

[4] Zhao, M.; Lin, J.; Wang, X.; Liao, Y. (2011). Dynamic transmission error analysis for a CNC machine tool based on built-in encoders, 2011 IEEE International Symposium on Assembly and Manufacturing (ISAM),5 pages, doi:10.1109/ISAM.2011.5942325

[5] Koren, Y. (2007). Control of machine tools, Journal of Manufacturing Science and Engineering, Vol. 119, No. 4B, 749-755, doi:10.1115/1.2836820

[6] Ibaraki, S.; Sawada, M.; Matsubara, A.; Matsushita, T. (2010). Machining tests to identify kinematic errors on five-axis machine tools, Precision Engineering, Vol. 34, No. 3, 387-398, doi:10.1016/j.precisioneng.2009.09.007

[7] Delbressine, F. L. M.; Florussen, G. H. J.; Schijvenaars, L. A.; Schellekens, P. H. J. (2006). Modelling thermomechanical behaviour of multi-axis machine tools, Precision Engineering, Vol. 30, No. 1, 47-53, doi:10.1016/j.precisioneng.2005.05.005

[8] Sharif Uddin, M.; Ibaraki, S.; Matsubara, A.; Matsushita, T. (2009). Prediction and compensation of machining geometric errors of five-axis machining centres with kinematic errors, Precision Engineering, Vol. 33, No. 2, 194-201, doi:10.1016/j.precisioneng.2008.06.001

[9] Slocum, A. H. (1992). Precision Machine Design, Society of Manufacturing Engineers, Dearborn

[10] Schwenke, H.; Knapp, W.; Haitjema, H.; Weckenmann, A.; Schmitt, R.; Delbressine, F. (2008). Geometric error measurement and compensation of machines - An update, CIRP Annals Manufacturing Technology, Vol. 57, No. 2, 660-675, doi:10.1016/j.cirp.2008.09.008

[11] Jung, J.-H.; Choi, J.-P.; Lee, S.-J. (2006). Machining accuracy enhancement by compensating for volumetric errors of machine tool and on-machine measurement, Journal of Materials Processing Technology, Vol. 174, No. 1-3, 56-66, doi:10.1016/j.jmatprotec.2004.12.014

[12] Chen, J. S.; Kou, T. W.; Chiou, S. H. (1999). Geometric error calibration of multi-axis machines using an auto-alignment laser interferometer, Precision Engineering, Vol. 23, No.4, 243-252, doi: $10.1016 / \mathrm{S} 0141-6359(99) 00016-1$ 
[13] Barakat, N. A.; Elbestawi, M. A.; Spence, A. D. (2000). Kinematic and geometric error compensation of a coordinate measuring machine, International Journal of Machine Tools and Manufacture, Vol. 40, No. 6, 833-850, doi:10.1016/S0890-6955(99)00098-X

[14] Bohez, E. L. J. (2002). Five-axis milling machine tool kinematic chain design and analysis, International Journal of Machine Tools and Manufacture, Vol. 42, No. 4, 505-520, doi:10.1016/S0890-6955(01)00134-1

[15] Wang, J.-D.; Guo, J.-J. (2012). Research on volumetric error compensation for NC machine tool based on laser tracker measurement, Science China Technological Sciences, Vol. 55, No. 11, 3000-3009, doi:10.1007/s11431-012-4959-6

[16] Okafor, A. C.; Ertekin, Y. M. (2000). Derivation of machine tool error models and error compensation procedure for three axes vertical machining centre using rigid body kinematics, International Journal of Machine Tools and Manufacture, Vol. 40, No. 8, 1199-1213, doi:10.1016/S0890-6955(99)00105-4

[17] Hughes, B.; Forbes, S.; Lewis, A.; Sun, W.; Veal, D.; Nasr, K. (2011). Laser tracker error determination using a network measurement, Measurement Science and Technology, Vol. 22, No. 4, Paper 045103, doi:10.1088/0957-0233/22/4/045103

[18] Liu, Y.; Gao, D.; Lu, Y. (2015). Volumetric calibration in multi-space in large-volume machine based on measurement uncertainty analysis, International Journal of Advanced Manufacturing Technology, Vol. 76, No. 9, 1493-1503, doi:10.1007/s00170-014-6367-5

[19] Ruiz-Arenas, S.; Horvath, I.; Mejia-Gutierrez, R.; Opiyo, E. Z. (2014). Towards the maintenance principles of cyber-physical systems, Strojniski vestnik - Journal of Mechanical Engineering, Vol. 60, No. 12, 815-831, doi:10.5545/sv-jme.2013.1556

[20] Novak, B.; Babnik, A.; Mozina, J.; Jezersek, M. (2014). Three-dimensional foot scanning system with a rotational laser-based measuring head, Strojniski vestnik - Journal of Mechanical Engineering, Vol. 60, No. 11, 685-693, doi:10.5545/sv-jme.2014.1950

[21] Chen, X.; Zhang, G. X.; Zhao, S. Z.; Duan, F. J. (2006). Analysis and measurement for the optical error of the cat's eye retro-reflector, Journal of Physics: Conference Series, Vol. 48, 6468, doi: $10.1088 / 1742-6596 / 48 / 1 / 012$

[22] Aguado, S.; Samper, D.; Santolaria, J.; Aguilar, J. J. (2012). Towards an effective identification strategy in volumetric error compensation of machine tools, Measurement Science and Technology, Vol. 23, No. 6, Paper 065003, doi:10.1088/0957-0233/23/6/065003

[23] Acko, B.; Godina, A. (2005). Verification of the conventional measuring uncertainty evaluation model with Monte Carlo simulation, International Journal of Simulation Modelling, Vol. 4, No. 2, 76-84, doi:10.2507/IJSIMM04(2)3.039

[24] Dotcheva, M.; Millward, H. (2008). A generation of more efficient CNC tool paths using simulation modelling, International Journal of Simulation Modelling, Vol. 7, No. 3, 135-145, doi:10.2507/IJSIMM07(3)3.108

[25] Linares. J. M.; Chaves-Jacob, J.; Schwenke. H.; Longstaff, A.; Fletcher. S.; Flore, J.; Uhlmann, E.; Wintering, J. (2014). Impact of measurement procedure when error mapping and compensation a small CNC machine using a multilateration laser interferometer, Precision Engineering, Vol. 38, No. 3, 578-588, doi:10.1016/j.precisioneng.2014.02.008

[26] Conte, J.; Majarena, A. C.; Aguado, S.; Acero, R.; Santolaria, J. (2016). Calibration strategies of laser trackers based on network measurements, International Journal of Advanced Manufacturing Technology, Vol. 83, No. 5, 1161-1170, doi:10.1007/s00170-015-7661-6

[27] Muralikrishanan, B.; Lee, V.; Blackburn, C.; Sawyer, D.; Phillips, S.; Ren, W.; Hughes, B. (2013). Assessing ranging errors as a function of azimuth in laser trackers and tracers, Measurement Science and Technology, Vol. 24, No. 6, Paper 065201, doi:10.1088/0957$\underline{0233 / 24 / 6 / 065201}$ 